\title{
Nonlinear space-time information processing
}

\section{Y. Fainman, Daniel Marom, Kazutaka Oba, Dmitriy Panasenko, Yuri Mazurenko, et al.}

Y. Fainman, Daniel M. Marom, Kazutaka Oba, Dmitriy Panasenko, Yuri T. Mazurenko, P. C. Sun, "Nonlinear space-time information processing," Proc. SPIE 10296, 1999 Euro-American Workshop Optoelectronic Information Processing: A Critical Review, 1029604 (2 June 1999); doi: 10.1117/12.365914

SPIE Event: Euro-American Workshop on Optoelectronic Information Processing, 1999, Colmar, France 


\title{
Nonlinear space-time information processing
}

\author{
Y. Fainman, D. M. Marom, K. Oba, D. Panasenko, Y. T. Mazurenko, and \\ P. C. Sun \\ University of California, San Diego \\ Department of Electrical and Computer Engineering \\ La Jolla, California 92093-0407
}

\begin{abstract}
Optical information processing, traditionally employed in the spatial domain, has been experiencing a renaissance with femtosecond laser pulse technology. Temporal optical information can now be manipulated via linear and nonlinear processes, and stored and retrieved, by converting optical signals between the spatial and temporal domains. In this manuscript, we review the state-of-the-art in the spatio-temporal optical signal processing techniques for information data coding, data conversion, signal recording, as well as signal characterization. Applications of these techniques for future computing, communication, storage, and signal processing systems are discussed.
\end{abstract}

\section{INTRODUCTION}

Ultrashort pulse laser technology has recently experienced significant advances, producing high peak power pulses of optical radiation few femtoseconds in duration, corresponding to only a few cycles of its fundamental frequency. ${ }^{1-4}$ Future progress in this area is inevitable due to the unique properties of ultrashort laser pulses that are crucial for various science and engineering applications including optical communications, ${ }^{5,6}$ medical and biomedical imaging, ${ }^{7-11}$ chemistry and physics. ${ }^{12-14}$ A common feature of these applications relies on our ability to control the shape of the ultrashort pulses as well as, conversely, our ability to detect the shape of the ultrashort pulses.

In communication applications, femtosecond laser pulse technology may enable efficient utilization of the available bandwidth of fiber optic communication systems. Presently, we are far from realizing the potential capacity of these networks. Electronic devices and systems, with their typical $1-10 \mathrm{~Gb} / \mathrm{s}$ rates, interconnected by photonic networks whose maximum bit-rate capacity may exceed $1 \mathrm{~Tb} / \mathrm{s}$ are the norm. The 2-3 order-of-magnitude mismatch between fiber and electronic device capacities can be used to increase the speed, reduce the latency, or increase the security and reliability of the data transmission. To implement these applications, it will be necessary to construct an alloptical pre-processor at the transmitter and a post-processor at the receiver, which will perform multiplexing and demultiplexing, respectively. The multiplexer, performing space-to-time transformation will combine relatively slow but parallel electronic channels into an ultrahigh bandwidth serial fiber-optic channel (i.e., parallel-to-serial conversion), whereas the demultiplexer will perform the inverse time-to-space transformation for electronic detection (i.e., serial-to-parallel conversion).

With the exception of spectral hole burning and photon echo optical storage techniques, most of the 3-D storage systems achieve the high aggregate bandwidth by storing and accessing pages of spatial information bits. The pages of information in 3-D holographic memories are multiplexed in the volume, using phase ${ }^{17}$ and/or wavelength multiplexed encoding. ${ }^{18,19}$ Although such page oriented approach may be useful for local access, processing, and communication of images and image format data, it is very unlikely that page oriented optical memories can be easily integrated with distributed 
information systems utilizing ultrahigh bandwidth optical network systems. Parallel-toserial format conversion $\mathrm{L} / \mathrm{O}$ devices ${ }^{20,21}$ will need to be developed for integration of such page oriented storage systems with the communication network systems. Many applications will benefit from an optical holographic memory that will store and retrieve information in a format that is suitable for direct interface and transmission through an optical fiber network. With this approach, the spatial image information is converted to time domain sequence and stored as a spectral hologram. The information retrieved from the memory occurs in a time-sequenced format, suitable for direct transmission over an optical fiber network at rates exceeding 1 Tbits/sec.

In this review paper, we present our approach for realizing these systems. In Section 2 , various spatio-temporal processors for space-time conversion of optical data are discussed. Section 3 is devoted to short pulse storage and nonvolatile readout, while Section 4 describes efficient modulation schemes for asynchronous data networking applications. Experimental evidence as well as discussions addressing the limitations of the current techniques and potential solutions are discussed within these sections. Section 5 summarizes our results.

\section{SPATIO-TEMPORAL CONVERSION PROCESSES}

\subsection{Parallel-to-serial Multiplexer (PSM)}

The most promising technique to implement the PSM uses a spectral domain pulse shaper (PS). This device is based on spectral decomposition of an ultrashort transform limited laser pulse, modifying the pulse spectrum by using a 1-D fixed ${ }^{22}$ or programmable 23,24 spatial mask and recombining the modified temporal spectrum back into the time domain to form a shaped pulse. This technique has been shown to produce femtosecond shaped pulse trains with terahertz repetition rate, high resolution, and high fidelity..$^{25}$

Despite the capability of producing high quality shaped pulses, the existing spectral domain pulse shaping devices do not meet the speed requirements for ultra-high bandwidth optical communication applications. The pulse shaping devices rely on the speed of converting the spatial frequency content of the parallel spatial channels into the temporal frequency spectrum of an ultrashort pulse. The first devices used fixed spectral filters that require prior calculation and fabrication of Fourier transform computer generated holograms (CGH) corresponding to the parallel input channels. ${ }^{26}$ Performing such calculations and spectrum modulation in real time will require not only very fast computation, but also very fast $\mathrm{V} / \mathrm{O}$ bandwidth spatial light modulators (SLM) that can meet the spatial resolution and dynamic range of such spatial Fourier transform CGH. Liquid crystal SLMs ${ }^{23}$ and acousto-optic SLMs ${ }^{24}$ that are currently being used for such dynamic pulse shaping have limited speed, resolution, and dynamic range, limiting their potential use for ultra-high bandwidth optical communication applications.

We have addressed some of these difficulties by introducing an all-optical parallel-toserial processor that is capable of converting spatial optical signals directly into temporal shaped pulses with one-to-one correspondence ${ }^{20}$. Our approach is based on combining optical information processing of spectral holography ${ }^{27,28}$ with that of conventional spatial Fourier transform holography. Our processor, shown schematically in Fig. 1a, consists of two independent optical channels for carrying the temporal and the spatial information. The temporal information-carrying channel consists of a pair of gratings and a 4-F lens arrangement. The incident pulses are transformed by the input grating and the first lens into a temporal frequency spectrum distribution in space at the focal plane, while the second lens and the output grating perform the inverse transformation of the temporal spectrum distribution back to the time domain. The spatial information- 
carrying channel is a simple optical spatial Fourier transform arrangement consisting of the input image plane and a beamsplitter to share the second lens of the temporal channel. To achieve interaction between the spatial and temporal frequency information we use a real time holographic material in a degenerate four-wave mixing arrangement.

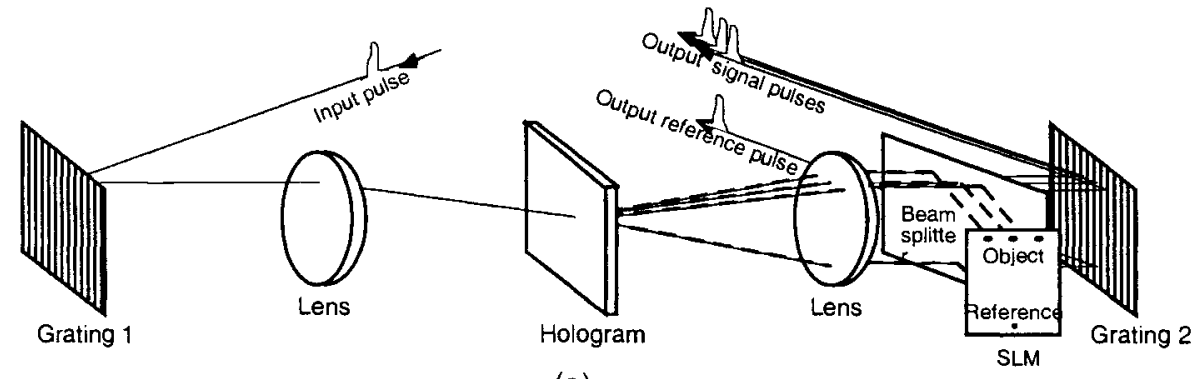

(a)

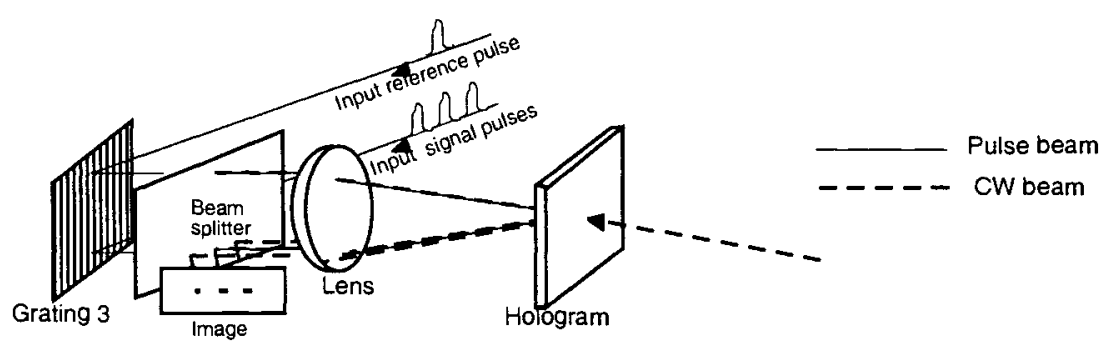

(b)

Fig. 1. Schematic diagram of optical processors for (a) parallel-to-serial conversion and (b) serial-to-parallel conversion.

For our initial experiment we used a 1-mm thick photorefractive crystal of $\mathrm{LiNbO}_{3}$. A 1-D binary input image (or a 1-D spatial light modulator) is illuminated by a monochromatic optical source and Fourier transformed onto the plane of the real-time holographic material where it interferes with the plane reference wave. The interference pattern is recorded via the photorefractive effect. The recorded spatial Fourier transform hologram is reconstructed by the temporal frequency spectrum of a femtosecond pulse with a center wavelength close to that of the monochromatic source used in the recording process. The temporal frequency spectrum is modulated by the spatial frequency information of the hologram. Upon transmission through the second lens and the output grating, the diffracted temporal frequency spectrum results in a sequence of short pulses which exhibit one-to-one correspondence with the 1-D spatial distribution in the input $\mathrm{CW}$ image. Note that the resultant sequence of temporal pulses is carried by a single beam, allowing easy coupling into an optical fiber link. For decoding of the temporal information at the receiver node we also need to transmit a single reference pulse.

At the receiver node we perform an inverse serial-to-parallel transformation. Such a transformation can be realized with spectral holography of the sequence of temporal pulses and a reference pulse as shown schematically in Fig. $1 b^{29,30}$ In the experiments we used $150 \mathrm{fsec}$ optical pulses at a wavelength of $480 \mathrm{~nm}$. During these experiments the output pulses from the system shown in Fig. la were transmitted directly to the input of the system shown in Fig. 1b. Two 1-D binary input images (see Fig. 2a) were used in our experiments for parallel-to-serial and serial-to-parallel conversion employing the 
processors shown in Fig. 1a and 1b, respectively. The received 1-D image in Fig. 2b shows exact correspondence to the original image in Fig. la.

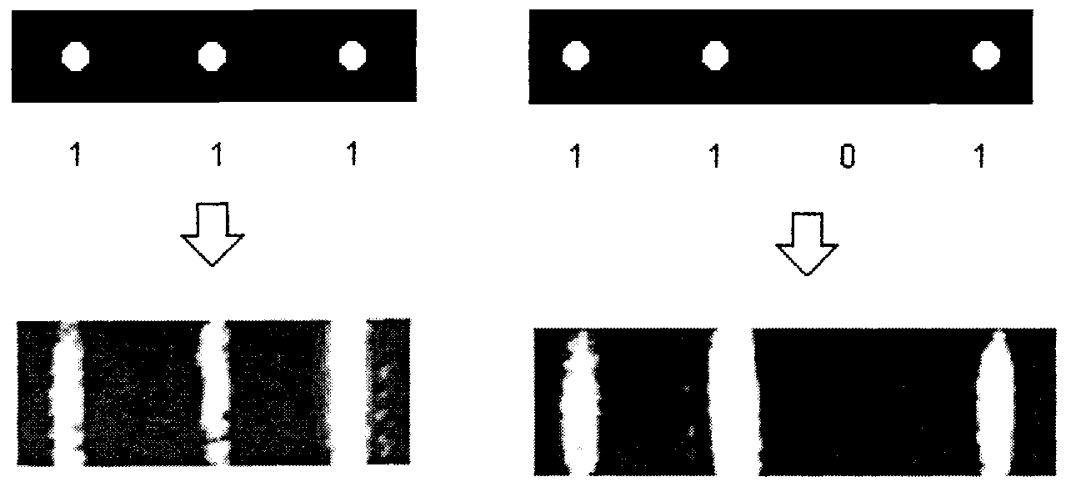

Fig. 2. Experimental result of image transmission using parallel-to-serial and serial-toparallel conversion: (a) the original 1-D image, (b) the received 1-D image

Photorefractive materials provide the necessary high resolution and wide dynamic range medium for recording and reconstruction of the spectral filters. In addition, with our approach the input signals are Fourier transformed optically into the spectral domain, thus alleviating the need for time-consuming electronic Fourier transform calculations used in the PS with programmable masks. The speed of this parallel-to-serial conversion is limited by the time response of the bulk photorefractive material, which, depending on the material, can vary in the range of seconds to microseconds. Recent studies of photorefractive multiple quantum well (MQW) devices ${ }^{31}$ decreased the time response to the sub-microsecond range, which is still far from meeting the speed requirements of ultra-high bandwidth optical communication applications. Furthermore, MQW devices will limit the conversion efficiency (to about $0.1 \%$ ) due to inherently small thickness, limiting the interaction length in the device. In summary, to meet the practical needs of the PSM system approach, it will be essential to increase the signal conversion speed and the conversion efficiency, which are the two main objectives of our current research. In the next section we present our preliminary findings towards achieving this goal.

\subsection{Spatial-temporal Four-wave Mixing using Cascade Second Order Nonlinearity}

To meet the speed requirements of the ultra-high bandwidth optical communication channel, the pulse-shaping device needs to be operated in real time, i.e., as fast as the time window of the time-multiplexed shaped pulse packet. Nonlinear optical processes such as nondegenarate four-wave mixing can achieve real time operation. Such nonlinear 4-wave mixing techniques usually suffer from low conversion efficiency due to relatively small values of $\chi^{(3)}$ in nonlinear optical materials. However, recent studies in Cascaded Second-order Nonlinearity (CSN) ${ }^{32-37}$ may provide a solution to this problem. The CSN is based on cascading two second-order nonlinear interactions in a second-harmonic generation experiment, resulting in accumulation of nonlinear phase matching of the fundamental field. The CSN approach has been used to achieve spatial soliton, ${ }^{32}$ selfdiffraction, ${ }^{33}$ as well as all-optical switching via optical Kerr effect, ${ }^{36,37}$ which are usually possible with third order optical nonlinearities. Since the normal phase-matching condition is no longer imposed by CSN, the conversion efficiency of this process can 
approach $100 \%$ when long interaction length is used. ${ }^{34}$ Recent experiments have demonstrated $15 \%$ conversion efficiency for CSN in a 1 -mm-thick BBO crystal. ${ }^{36}$

We report the first successful realization of the spatial-temporal processor capable of generating synthesized temporal waveforms at the output, controlled by a spatial domain image with femtosecond response time and high conversion efficiency. The CSN arrangement we are utilizing consists of a frequency-up conversion process followed by a frequency-down conversion process satisfying the type-II non-collinear phase matching condition. The non-linear wave mixing in our experiment takes place in the Fourier domain of the temporal and spatial channels (see Fig. 3). The first nonlinear process of the cascade mixes the spectral decomposition wave (SDW) field $U_{1}$ of an input ultrashort pulse denoted by $p(t)$ at the center frequency $\omega_{0}$ and the spatial FT field $U_{2}$ of a quasi-monochromatic wave of frequency $\omega_{0}$ spatially modulated by a one-dimensional

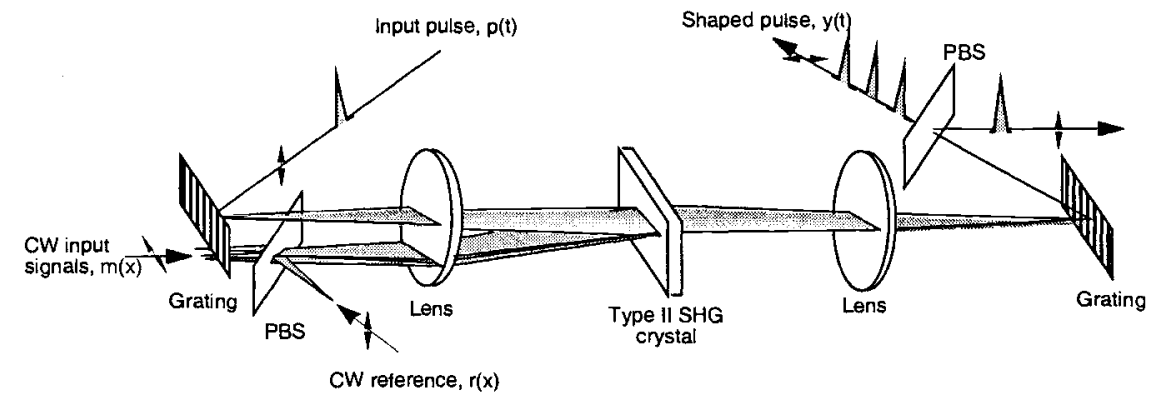

image denoted by $m(x)$.

Fig. 3. Experimental setup of the spatial-temporal processor. The CSN result in information exchange in a four-wave mixing process equivalent.

The fields $U_{1}$ and $U_{2}$ are polarized in the ordinary and extraordinary direction, respectively, relative to the optical axis of the nonlinear crystal and their propagation directions satisfy the phase matching condition. The waves $U_{1}$ and $U_{2}$ generate the intermediate up-converted wave $U_{i n t} \sim \chi^{(2)} U_{1} U_{2}$, oscillating at the sum frequency $2 \omega_{0}$, propagating along the optical axis of the setup, and polarized in the extraordinary direction. The second nonlinear process of the cascade mixes the intermediate wave $U_{\text {int }}$ and the spatial FT of a narrow slit $r(x)=\delta(x)$ (implementing a one-dimensional spatial Dirac $\delta$-function), $U_{3}$. The narrow slit in the second spatial channel is illuminated by the same quasi-monochromatic source as $U_{2}$, and is co-propagating with $U_{2}$ after a polarizing beam splitter (see Fig. 3). The wave $U_{3}$ is polarized in the ordinary direction and interacts with the extraordinary polarized intermediate wave $U_{\text {int. }}$. Since $U_{3}$ contains no information, in either the space or the time domain, its function is to down-convert the carrier frequency of the complex field of $U_{\text {iut }}$. In this arrangement, the phase matching condition for the down-conversion process is automatically satisfied. The resultant wave, $U_{4} \sim \chi^{(2)} U_{\mathrm{int}} U_{3}{ }^{*}$, propagates in the same directions as $U_{1}$ and at the same center frequency but in an orthogonal polarization state. The resultant wave is therefore proportional to the input waves as $U_{4} \sim\left(\chi^{(2)}\right)^{2} U_{1} U_{2} U_{3}^{*}$, which is equivalent to a four-wave mixing process.

Thus, the femtosecond rate spatial-temporal processing has generated the SDW of the output temporal optical waveform. The SDW $U_{4}$ is recombined in the optical setup by a second FT lens and grating diffraction to yield the output temporal signal, y $(\mathrm{t})=\mathrm{m}$ $(\mathrm{ct} / \alpha) * \mathrm{p}(\mathrm{t})$, where $\mathrm{c}$ is speed of light and $\alpha$ is the grating dispersion parameter $\alpha=\sin \theta$ with $\theta$ being diffraction angle. This synthesized waveform is a convolution of the input 
ultrashort pulse with a space domain image, whose spatial dependence has been converted to temporal dependence in the spatial-temporal processor. When the duration of the ultrashort pulse is much shorter than the feature size of the temporally mapped mask, then $p(t)$ can be approximated by a Dirac $\delta$-function and the output temporal waveform is directly proportional to the information in the mask. If the mask contains fine features, then the output waveform will be a smoothed version of the mask due to the convolution operation.

We demonstrate experimentally the CSN spatial-temporal wave mixing using ultrashort pulses of $100 \mathrm{fsec}$ duration at a center wavelength of $800 \mathrm{~nm}$ with energy level of $1 \mathrm{~mJ}$ per pulse (generated from a $\mathrm{Ti}$ : Sapphire ultrashort pulse oscillator combined with a regenerative amplifier). Ten percent of the power of the laser pulse was split off and introduced into the temporal input channel of the processor, generating the SDW $U_{1}$. The SDW is generated by a 600 lines $/ \mathrm{mm}$ blazed grating (dispersion parameter of $\alpha=0.48$ ) and a lens of $375 \mathrm{~mm}$ focal length. The remaining $90 \%$ of the pulse power were used to generate the light source for the implementation of the spatial channels by stretching the pulse width with a grating pair to several picosecond duration (i.e., chirped pulse), matching the time window of the temporal channel. The stretched pulse was divided into the two spatial channels for implementing waves $U_{2}$ and $U_{3}$. Since the CSN process occurs with femtosecond-scale time response due to the fast nonlinearity, a sufficient condition for four-wave mixing operation is that the two spatial channels be instantaneously equal. The spatial-temporal wave mixing by the $\chi^{(2)}$ media was performed in a $2-\mathrm{mm}$ thick type II $\beta$-barium borate (BBO) crystal. In our experiment, the entire process is derived from a single pulse from the laser source; thus, the information exchange is done on a single shot basis. Several experiments were conducted to illustrate this real-time processing technique, demonstrating its ability to control amplitude and phase in the output temporal waveform derived from the spatial information channel.

For initial characterization of the spatial-temporal wave mixing process via CSN, the two spatial channel waves were focused by cylindrical lenses to form line sources at the input spatial channels, generating plane waves $U_{2}$ and $U_{3}$ in the $\mathrm{BBO}$ crystal. The down-converted SDW $U_{4}$ was generated only in the presence of the waves from the two spatial channels. The maximum conversion efficiency of the input SDW $U_{1}$ to the filtered output SDW $U_{4}$ was $10 \%$, limited by fundamental wave depletion. The conversion efficiency depends on the power of the two spatial channel waves, as they serve as the pump waves in the CSN process. The high conversion efficiency illustrates the advantage of the CSN approach as opposed to conventional $\chi^{(3)}$ nonlinearity for fourwave mixing. In our first spatial-temporal information transfer experiment, we used a mask containing a sequence of narrow slits spaced $0.8 \mathrm{~mm}$ apart. To achieve high light throughput, the illuminating beam was focused into the slits with a cylindrical lenslet array. The shaped waveform, consisting of a sequence of pulses, was observed with a realtime pulse imaging technique (see Fig. 4). As predicted, the synthesized waveform consists of a sequence of pulses separated by $\sim 1.3$ psec (mapping spatial separation of 0.8 $\mathrm{mm}$ to time). Selectively blocking some of the slits resulted in a matching temporal waveform, confirming our ability to perform single shot temporal waveform synthesis in real-time from a spatial channel. These results were generated under maximal conversion efficiency, where fundamental wave depletion was observed. Therefore, by blocking some

of the slits, more photons are upconverted by the spatial waves of the open slits, leading to an amplitude distribution change in the pulse sequences of Fig. 4. No evidence of crosstalk between the channels was detected.

To demonstrate the ability to encode phase information, the spatial information channel consisted of a variable spherical wave front, generated by longitudinally translating a cylindrical focusing lens away from the input plane. The spatial-temporal 
information exchange converted the spatial quadratic phase to temporal quadratic phase, generating chirped pulses at the output of the processor. We estimated the amount of chirp using the pulse imager by the technique outlined in Ref. 38 (see also the description in the next section) and as expected we found direct correspondence between the longitudinal translation of the focusing lens and the amount of chirp on the synthesized waveform.
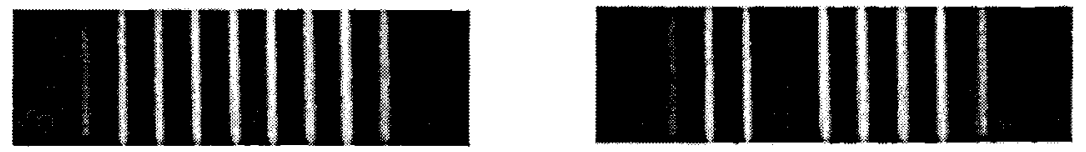

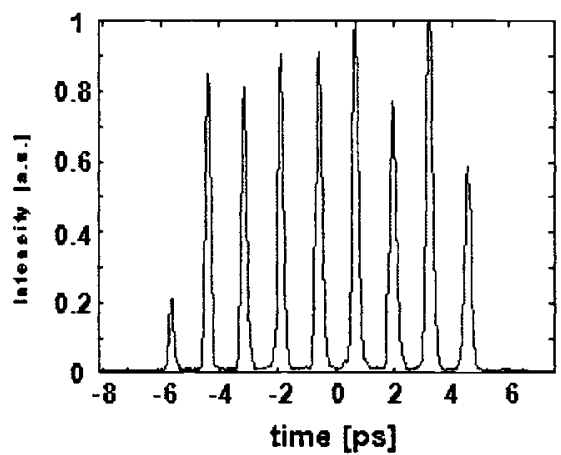

(a)

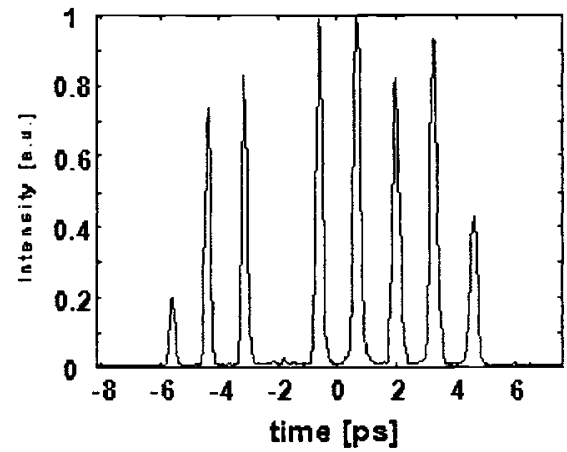

(b)

Fig. 4. Synthesized temporal waveform generated by a spatial information mask consisting of a sequence of equally spaced point sources. (a) All point sources are illuminated by quasimonochromatic light. (b) One point source blocked.

Relative to other spatial-temporal processing techniques, the CSN approach provides femtosecond rate processing due to the fast bound electron nonlinearity and high efficiency on account of a relatively large $\chi^{(2)}$ coefficient. The spatial-temporal process that we have demonstrated generates an output temporal waveform that can be reconfigured in real time and is proportional to the convolution of an input ultrashort pulse and a spatial image. Furthermore, wavelength tuning of the synthesized temporal waveform can be achieved by using different temporal frequencies in the two spatial channels (with a correction to the propagation direction, to satisfy phase matching). For operation with pulsed lasers at high repetition rates, the spatial channels may be implemented by a second intense $\mathrm{CW}$ laser source or the conversion efficiency of the CSN process should be improved. Since the technique realizes a general four-wave mixing process of temporal and spatial information-carrying waves, the setup may be converted to provide the convolution or correlation signal between spatial and temporal channels, with the output in either the temporal or the spatial domain. Thus, this spatial-temporal process can be considered a fundamental system for performing ultrafast signal processing on optical waveforms in the time and space domain.

\subsection{Pulse Image Converter (PIC)}

The ultrafast pulse image converter performs serial-to-parallel demultiplexing of the shaped pulse train created by the pulse shaper back into parallel spatial domain for electronic detection. The speed requirement of PIC is even higher than PS, since the conversion speed needs to match the bit rate of the temporal signal generated by the PS (in the range of THz). The purpose of the PIC is to "slow down" the pulse signals by 
48 / Critical Reviews Vol. CR74

converting the signals into parallel channels where each channel possess a reduced bit rate of $10-100 \mathrm{GHz}$ that is within the operational bandwidth of electronic detectors. Therefore, the concern here is how to achieve an optical serial-to-parallel conversion at such high speed of operation.

Since the photorefractive four-wave mixing approach suffers from slow time response and does not meet the speed requirements of the demultiplexer, we have demonstrated an alternative approach based on nonlinear three-wave mixing that overcomes the speed limitations of photorefractives. Our PIC system ${ }^{38}$ is capable of realtime conversion of a femtosecond pulse sequence into its spatial image. The approach employs nonlinear spectral domain 3-wave mixing in a crystal of $\mathrm{LiB}_{3} \mathrm{O}_{5}$ (LBO), where the SDW of a shaped femtosecond pulse are mixed with those of a transform limited pulse to generate a quasi-monochromatic second harmonic field. Through this nonlinear process, the temporal frequency content of the shaped pulse is directly encoded onto the spatial frequency content of the second harmonic field, producing a spatial image of the temporal shaped pulse.

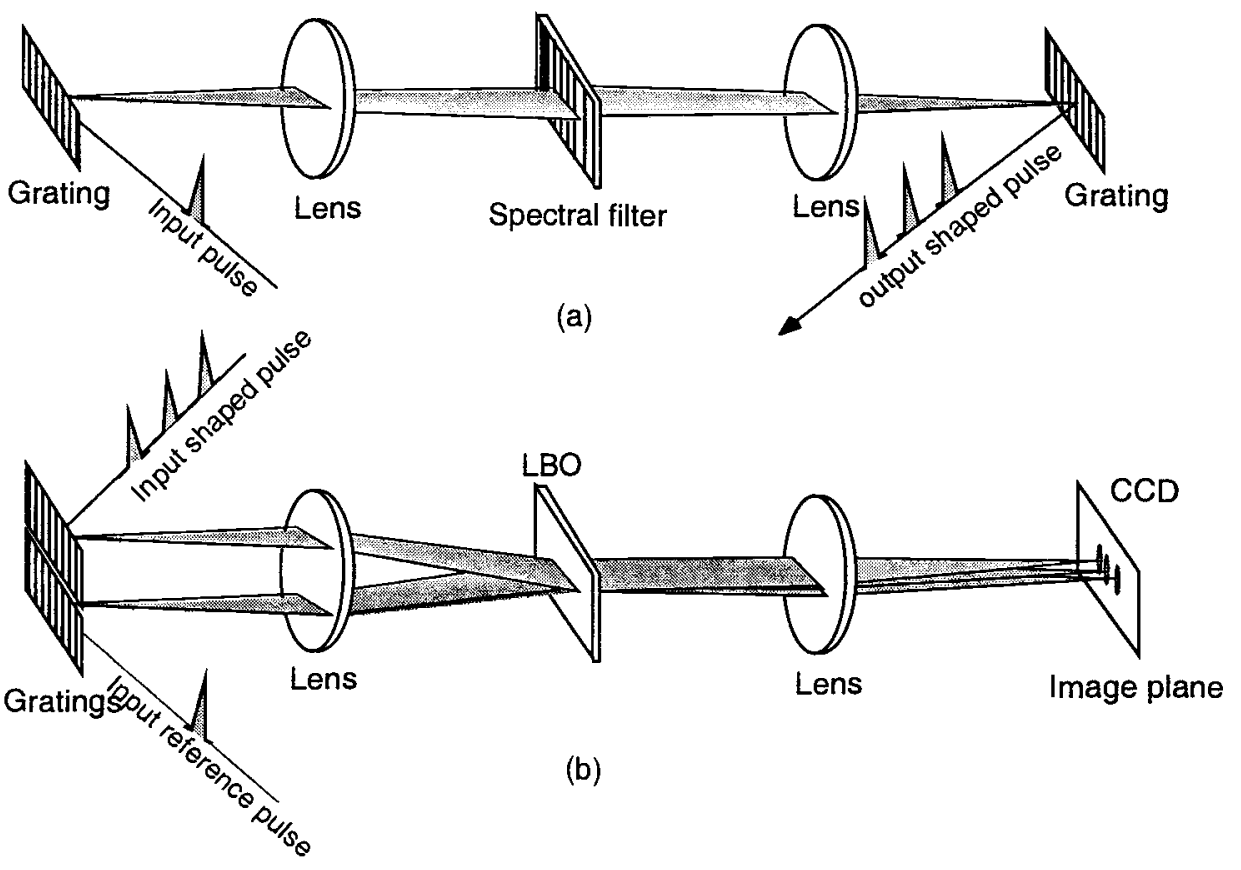

Fig. 5. Schematic diagram of (a) the pulse shaping device with a spectral domain spatial filter and (b) the femtosecond pulse imaging system based on nonlinear spectral domain 3wave mixing in LBO crystal.

The femtosecond laser output is split into two beams, one to be used as a reference beam and the other sent into a pulse shaping device (see Fig. 5a) to create a shaped pulse. The shaped pulse and the reference pulse beams are then introduced into the pulse imaging system of Fig. 5b. The two incident beams arrive in opposite directions, in order to obtain the necessary spectrum inversion of the corresponding SDW. The beams are vertically displaced to satisfy the non-collinear phase matching condition. These two beams are introduced into a LBO nonlinear crystal, generating a second harmonic field that 
propagates in a bisector direction that is parallel to the optical axis of the system. A second lens is used to perform a spatial Fourier transform of the second harmonic quasimonochromatic field, producing an image that is detected by a CCD camera. ${ }^{38}$

In our experiments, we use a 50/50 binary amplitude grating (i.e., a Ronchi grating) as a spectral filter in the pulse-shaping device. Such a grating has a unique property that its Fraunhofer diffraction pattern does not have even diffraction orders except the zero-th order. The pulse image (see Fig. 6) clearly shows that the even order pulses in the sequence (except the 0 -th order) do not appear. The three central pulses (i.e., $-1,0,+1$ orders) are close together while the other pulses (corresponding to higher orders) are separated by twice the distance. We estimate the distances between the center pulses to be 1.75 picoseconds which is consistent with the theoretical prediction.

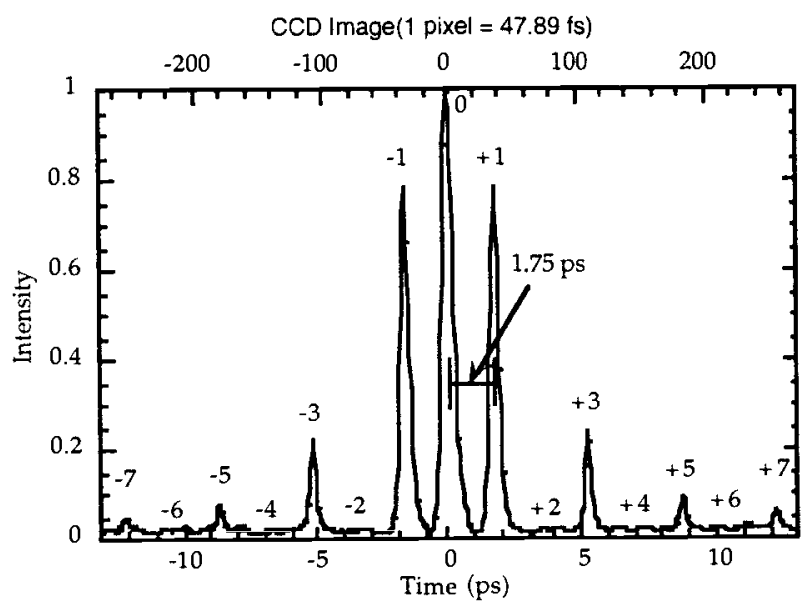

Fig. 6. The images of shaped pulses obtained with femtosecond pulse imaging system of Fig. 5. The shaped pulses are obtain using a pulse-shaping device with spectral filter implemented by a Ronchi grating.

In the PIC experiments we use a Ti: Sapphire laser that produces 200 femtosecond pulses of $10 \mathrm{~nJ}$ per pulse at a rate of $77 \mathrm{MHz}$. The measured conversion efficiency of the 3 -wave mixing is relatively low $(0.1 \%)$ due to the spatial chirping of the pulse that reduces the peak power density essential for nonlinear wave mixing in the LBO crystal. However, our experiments show that employing $200 \mathrm{fsec}$ pulses with 1-10 nJ energy per pulse, we can generate image intensity of the second harmonic field that is sufficient for CCD detection with video rate integration time. For PSM application, the integration time will need to be reduced to nanosecond range, which in turn will require improvement in conversion efficiency of the second harmonic field for reliable signal detection. For practical applications we need to increase the conversion efficiency at least by one order of magnitude.

Currently, there are two new techniques that can help increasing the conversion efficiency. (i) The first technique uses periodically poled nonlinear crystals (e.g., $\mathrm{LiNbO}_{3}$ ) to create a quasi-phase-matched condition for second harmonic generation over a long interaction length..$^{39.41}$ A quasi-phase-matching condition can be satisfied for a much longer interaction length in a nonlinear optical three-wave mixing by flipping periodically the domain polarity of the bulk $\mathrm{LiNbO}_{3}$ with periodicity matching the coherence length. As a result the quasi-phase matching causes an increase in the effective interaction length 
resulting in a dramatic improvement in the conversion efficiency. This technique has been demonstrated to produce very high efficiency for three-wave mixing for frequencydoubling, sum-frequency generation, and difference frequency mixing. (ii) The second technique is based on enhancing optical nonlinearity by using microstructured composite nonlinear optical materials. ${ }^{42-43}$ The composite material is formed by combining into one system two or more optical nonlinear and/or linear materials using periodic microstructures (e.g., multilayers, grating structures, etc.). The periodicity in such composite materials is much less than the wavelength of the radiation, such that the material behavior is similar to that of a bulk material, but, with its optical properties far different from any of its constitute components. With proper design of the structure geometry, the optical nonlinearity of the composite material can be enhanced. About one order of magnitude enhancement is expected over the nonlinearities of the constituent materials. ${ }^{43}$ The enhancement is due to the local field effects, which can be understood as a local field concentration resulting from the index periodicity of the structure.

\section{NONVOLATILE STORAGE OF A SEQUENCE OF ULTRASHORT PULSES}

Many application may benefit from an optical holographic memory that will store and retrieve information in a format that is suitable for direct interface and transmission through an optical fiber network, thereby providing optimal performance in terms of hardware complexity, memory and network capacity bandwidth, and latency. With such approach the spatial image information is converted to time domain sequence and stored as a spectral hologram. These conversion devices must at least preserve the aggregate bandwidth of the storage system or even multiplex few storage systems to meet and utilize the ultrahigh bandwidth of the evolving optical communication network systems. At the receiver node of the network, the time sequence data can be demultiplexed by converting the time sequence back to the parallel spatial channel for electronic detection, processing and display. In this section, we will introduce two different approaches for storage of a sequence of ultrashort pulses. In particular, we will focus on spectral holographic approach suitable for optical communication system applications.

\subsection{Time Domain Storage}

Time domain storage ${ }^{44-46}$ is based on direct recording and reconstruction of timedependent or space-time-dependent optical signals. An important application of timedomain holography is 3-D volume holographic storage. The existing 3-D volume holographic memory systems store pages of spatial information using phase ${ }^{17}$ and/or wavelength multiplexed encoding ${ }^{18,19}$. During readout, an appropriate code is set onto the reference beam that reconstructs the corresponding page of information at the output of the memory system. In contrast to the existing photorefractive 3-D memories, a new approach uses true 3-D storage, where the pages of information are stored in separate subvolumes. Such partitioning of the volume can be obtained using a technique based on collision of ultra-short pulses. ${ }^{47}$

An ultra-short laser pulse is split into two, where one is modulated by the 2-D NxN spatial image information that is to be stored, while the other is used as a reference wave to record a reflection-type hologram. The information carrying pulse is imaged into the volume of the photorefractive media where it collides with the counter-propagating uniform amplitude reference pulse in the volume of the holographic material centered at a single longitudinal coordinate $\mathrm{z}_{\mathrm{i}}$. This coordinate is determined by the relative time delay between the two beams. The intensity distribution of the interference between the collided pulses will give rise to recording a reflection-type volume hologram, confined in 
1999 Euro-American Workshop on Optoelectronic Information Processing / 51

the longitudinal direction. The dimension of this confinement is determined by collision length, i.e., by the spatial extend of the pulse, $l_{p}=c / t_{p} n$, where $c$ is the speed of light in vacuum, $t_{p}$ is the pulse duration, and $n$ is the refractive index of the volume holographic material.

For retrieval of the recorded information (memory readout), the reference pulse of duration $t_{p}$, is sent into the volume hologram which diffracts on the recorded subvolumes generating a sequence of time-delayed 2-D pages of the recorded information images. To access a single page from the reconstructed sequence one must use a decoding technique, which for the case of time-space coding uses existing time-gating techniques. ${ }^{48}$ The random access to the pages in the sequence can be accomplished using either acousto-optic or electro-optic devices integrated with discrete temporal delay lines. For example, with an electro-optic or acousto-optic deflector of $\mathrm{t}_{\mathrm{a}}=1 \mu \mathrm{sec}$ and pages of size $\mathrm{N}^{2}=10^{6}$, we can obtain data transfer rates of $1 \mathrm{Tbit} / \mathrm{sec}$.

Direct time domain holography suffers from low fringe contrast and limited time window. Fringe contrast is determined by the cross-correlation function between the signal and the reference wave packets, which decreases for the signal wave packets containing a long pulse train sequence. The overall time window for direct time domain holography depends on the dimension of the recording medium (in the pulse propagation direction), but not on the duration of the pulse. For example a temporal sequence of 1000 pulses of 1 psec each will occupy a space of $30 \mathrm{~cm}$, which is much longer than the thickness of available volume holographic photorefractive materials (typically $1 \mathrm{~cm}$ thick), thereby only a fraction of the 1000 pulses can be recorded. These two disadvantages of time domain holography can be overcome by using spectral domain holography.

\subsection{Spectral Domain Holography}

The spectral holography approach $27,28,49,50$ uses the temporal spectrum decomposition of the signal wave packets. The spectrum decomposition process can be accomplished by using an optical spectral device built of a grating or a prism in combination with a lens. The SDW of a short wave packet from such a spectral device is linearly dispersed along the $\mathrm{x}$-direction, where each sub-division of the wave in the $\mathrm{x}$ direction contains a longer wave packet of narrower spectral bandwidth compared with the temporal extent of the original wave packet. If two SDW, one from the signal wave packet and the other from the reference wave packet, are brought together in a volume of the recording photorefractive medium, a spectral hologram is formed. During the reconstruction process a spectral device is used to convert the information of the spectral hologram back to the time domain. Due to the narrower spectral bandwidth of the spectral decomposition waves dispersed in space, the fringe pattern and the recorded spectral hologram will have much higher contrast in comparison to that obtained with direct time domain holography. Improvement in the fringe contrast is achieved by utilizing only one dimension for the spectral holographic recording. To record multiple wave packets, shift multiplexing can be utilized, resulting in a recording format over the entire volume of the holographic material. The time window of spectral holography depends on the resolution of the optical spectral decomposition device. If a longer recording time period is desired, then it is necessary to choose a higher resolution spectral decomposition device such as a Fabry-Perot etalon. Experimental results ${ }^{51}$ have shown a wave packet of nanosecond duration recorded and reproduced by a spectral hologram when Fabry-Perot etalons are employed as spectral decomposition devices. The wave packets of such duration correspond to a few tenths of centimeters in space and are not practical for recording with direct time domain holographic techniques. 
The information storage time is an important issue associated with using photorefractive materials for volume holographic storage applications. If the same wavelength is used for recording as well as for reconstruction of a photorefractive hologram, then the reconstruction process will necessarily cause eraser of the recorded information hologram. This issue has been addressed by using different wavelength of radiation during the recording and reconstruction of the information holograms. The reconstruction is performed at a wavelength for which the sensitivity of the photorefractive material is essentially zero, provided the Bragg matching condition at the reconstruction wavelength is satisfied to obtain efficient readout. Several elaborate solutions to the Bragg matching condition have been proposed in page oriented holographic memory systems where dual wavelength recording and reconstruction have been employed..$^{52}$ To achieve nonvolatile storage, we use dual wavelength recording and reconstruction of spectral holograms. Our technique does not require any special arrangement to satisfy the Bragg matching condition. It can be satisfied over the entire spatial frequency range by adjusting the angle of incidence of the spectral decomposition of the readout wave. Such simple adjustment is possible because holograms recorded with our method have a quasi-1D data format and we can choose the direction of the spatial carrier to be orthogonal to the direction of the wavelength decomposition.

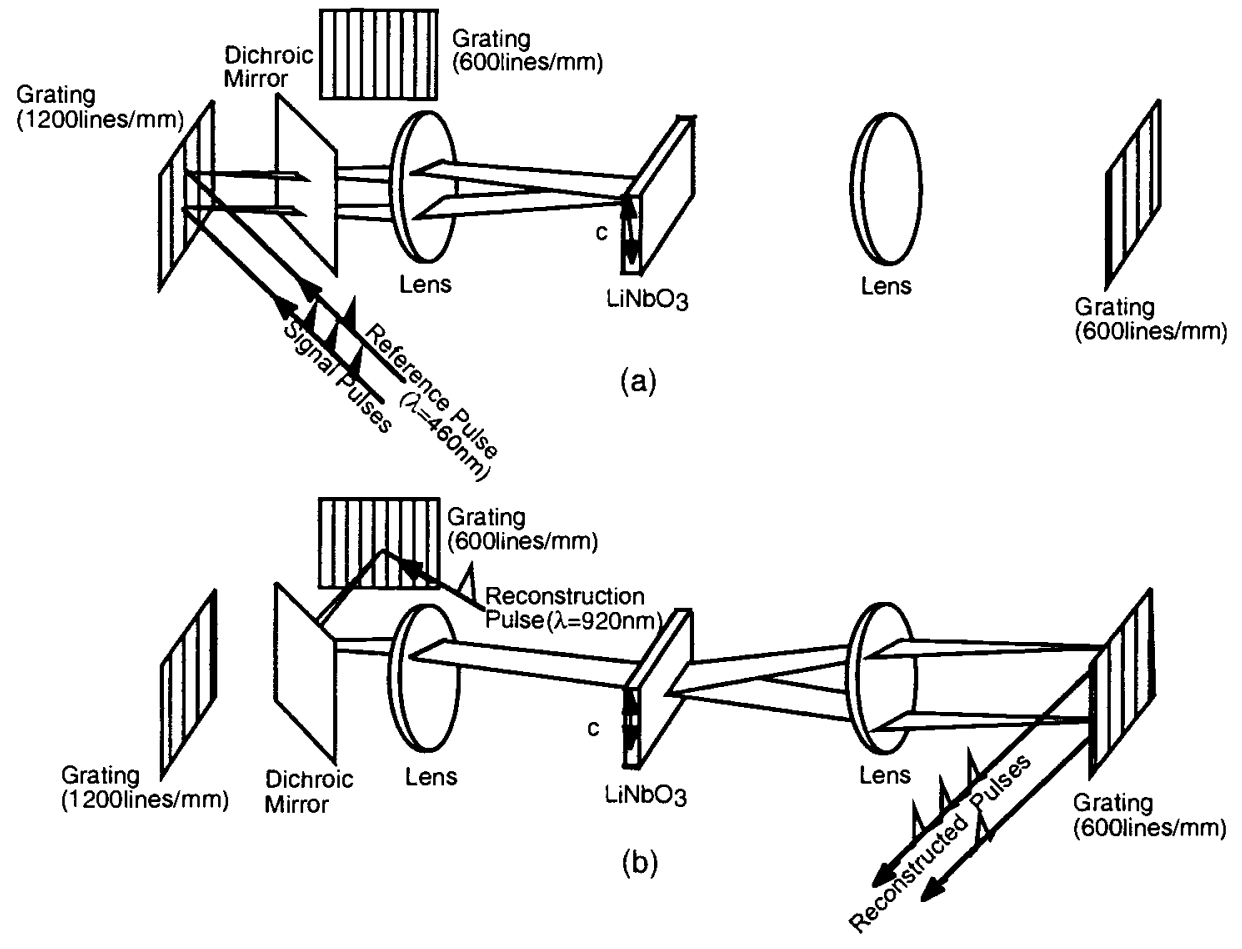

Fig. 7. Time-domain storage system using spectral holographic recording. (a) recording setup (b) reconstruction setup.

The experimental set-up for spectral holographic storage is schematically shown in Fig. 7. We use a mode-locked Ti: Sapphire laser producing 200 fs pulses at the center wavelength of $920 \mathrm{~nm}$ with a repetition rate of $77 \mathrm{MHz}$. The infrared radiation $(920 \mathrm{~nm})$ is divided into two beams, one which is kept for the hologram reconstruction process while the other is frequency-doubled using a second harmonic generator producing pulses 
at a center wavelength of $460 \mathrm{~nm}$ for hologram recording. The radiation at $460 \mathrm{~nm}$ is split into two beams for the recording process, one used as a reference beam while the other one is transmitted through a pulse shaper to produce an object beam pulse sequence. The pulse sequence is tailored using spectral domain filtering of an incident transformlimited pulse introduced into a pulse shaper.

The object beam from the pulse shaper and the reference pulse are introduced into the spectral holographic storage system of Fig. 7(a). Both beams propagate parallel to each other but are separated vertically, introducing a spatial carrier for recording the spectral hologram. Both beams diffract from the first reflection grating of 2400 lines $/ \mathrm{mm}$ and are spatially Fourier transformed by the first lens. The SDW components of both the signal pulse and the reference pulse are identically dispersed in the horizontal direction while in the vertical direction they merge and overlap each other. The spectral components from both beams coincide, producing an interference pattern that can be recorded as a spectral hologram. A $1-\mathrm{mm}$ thick $\mathrm{LiNbO}_{3}$ crystal is placed in the Fourier transform plane for recording of this spectral hologram. Typical recording times vary in an interval of $60 \mathrm{sec}$ to $90 \mathrm{sec}$. As the recording process takes a relatively long time, the stability of the system is crucial.

During the reconstruction process, a readout pulse beam of $920 \mathrm{~nm}$ wavelength is introduced into the recording system via a dichroic beam splitter. The readout beam, after diffracting from a reflection grating of 600 lines $/ \mathrm{mm}$, is then Fourier transformed by the first lens. To satisfy the Bragg matching condition, the readout beam is shifted vertically to achieve the best diffraction efficiency from the spectral hologram. In our experiment, we set the scaling factor to 1 by choosing gratings with appropriate spatial frequencies. The reconstructed beam from the hologram is transmitted through the second Fourier transform lens and combined by the second reflection grating of $600 \mathrm{line} / \mathrm{mm}$. The reconstructed pulses are introduced into a cross-correlator for detection and analysis.

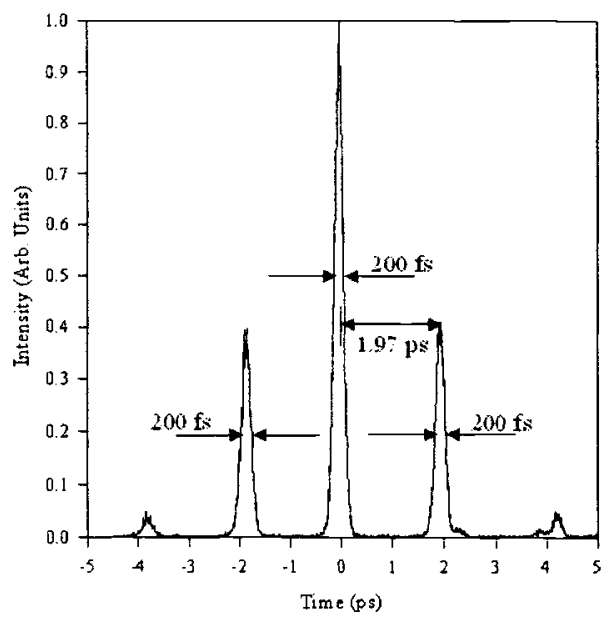

Fig. 8.. Experimental result from Setup of Fig. 7.

Figure 8 shows the experimental results of the reconstructed pulse sequence that was stored in the spectral hologram. The reconstruction result shows that each of the reconstructed pulses has a pulse width of about $200 \mathrm{fs}$ and that the pulse separation is $1.97 \mathrm{ps}$, consistent with the calculated pulse separation of $2.1 \mathrm{ps}$ with the scaling factor of about 1 . The diffraction efficiency is measured to be $7 \%$, and did not show any 
54 / Critical Reviews Vol. CR74

degradation for over 24 hours of continuous reconstruction. The second order peaks appeared due to imperfections in the Ronchi grating used in our experiment.

For spectral holography with dual-wavelengths used in writing and reading the hologram, the time separation of the readout pulse train can be larger or smaller than that of the input recorded pulse train. This is similar to the effect of magnification in conventional spatial holography when different wavelengths are used during the writing and reading the hologram. The reconstructed image can be magnified or de-magnified depending on the ratio between the writing/reading wavelengths. In contrast, the magnification in spectral holography is also affected by the spectral resolving power of the gratings used during the recording and reconstruction processes. For example, it is possible to record a spectral hologram of nanosecond pulse signals and reconstruct them with sub-picosecond scale pulses, obtaining nearly 1000 times magnification. For this application, a high spectral resolution device, such as a Fabry-Perot interferometer ${ }^{51}$ is required for spectral decomposition of the nanosecond pulses.

\section{PULSE PROCESSING, ENCODING AND DECODING}

\subsection{Recall, Time Reversal, and Temporal Filtering of Optical Pulses}

Similar to spatial holography that allows us to reconstruct the real and virtual images of an optical signal, the reconstruction of a spectral hologram can generally reproduce two signals: the original signal wave packet and the time-inverted signal wave packet. These two output signals represent the real signal and the phase-conjugate signal reconstructed from the hologram. In addition to the ability to record and reconstruct optical wave packets, spectral holography is also capable of performing filtering of optical wave packets in the time domain ${ }^{28,49,50}$. This concept is similar to that of Vander Lugt filtering for spatial domain filtering. Consider a set of target spatio-temporal signal wave packets that are pre-recorded in the form of spectral holograms multiplexed in the volume of a photorefractive material for later use as temporal filters. Let a test spatiotemporal signal wave packet be introduced into a temporal Fourier transform processing system containing the hologram with the multiplexed temporal filters. Depending on the orientation of the filters, the system can produce at the output either the convolution or the cross-correlation between the incident signal wave packet and one of the prestored target wave packet signals. Cross-correlation output is used for implementing temporal matched filtering, pulse compression, and dispersion compensation ${ }^{49,50}$. The combination of the convolution and cross-correlation has been used for temporal signal encoding and decoding 50 . Finally, volume spectral holograms have been used to control the shape of ultra-short laser pulses for ultrafast spectroscopy applications $s^{50}$.

\subsection{Hybrid Pulse Position Modulation and Code-division Multiple Access with Optical Short Pulse}

We are also investigating efficient modulation schemes in combination with ultrashort pulse communication. For asynchronous network applications, code division multiple access (CDMA) encoding of ultrashort pulses has been proposed ${ }^{15,16}$. A transmitted pulse is encoded by a filter applied to the spectrally dispersed wave, realizing a unique phase code. Since the encoding filter dephases the spectral content of the pulse, the temporal output signal is of long duration and low intensity, resembling a noise burst in its statistical properties. A decoding filter at the receiver, which matches only one encoded waveform, despreads the signal back to an ultrashort pulse form, while other encoded signals remain as low intensity background noise (see Fig. 9). To filter out the interference term, a precisely timed ultrashort time filter needs to select the decoded pulse 
for detection. Due to the unavailability of such temporal filters, nonlinear threshold detection schemes were suggested ${ }^{53}$ to distinguish the high peak power pulse against the low intensity interference. Using our pulse imaging apparatus to convert temporal information to spatial information, the filtering function can be performed by a simple slit. A detector placed behind the mask registers only desired filtered signal.

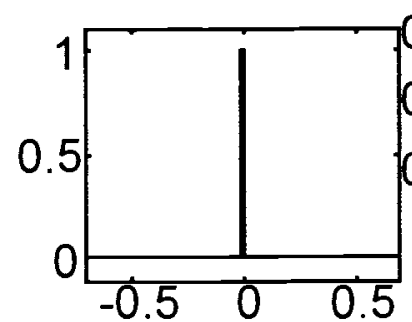

(a)

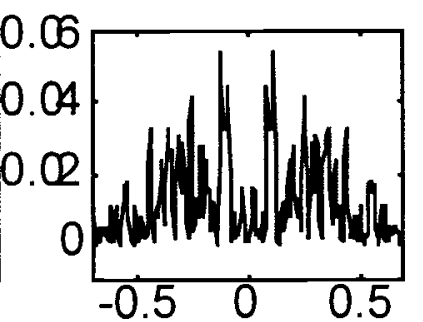

(b)

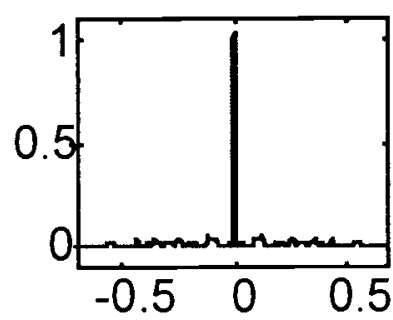

(c)

Fig. 9. (a) lnput short pulse form. (b) Encoded signal, with increased duration and noise burst properties. (c) Received signal in a network environment, after decoding the transmitted pulse.

Other users' signals appear as background noise.

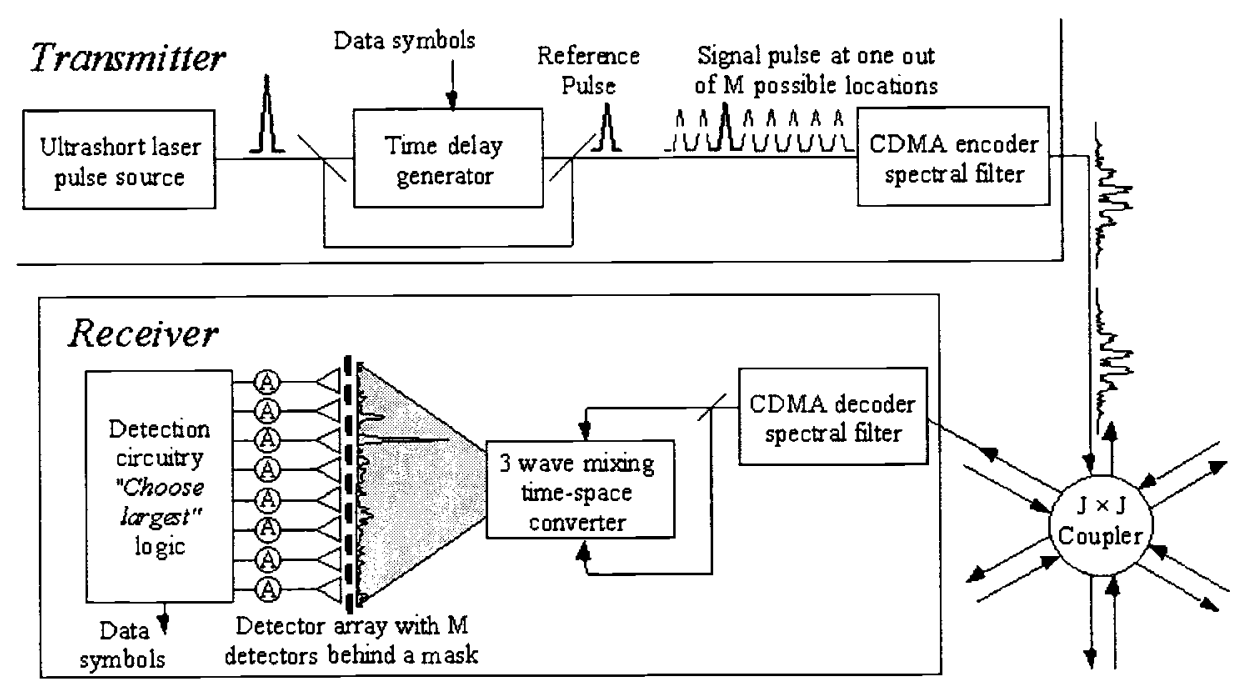

Fig. 10. System layout for hybrid PPM/CDMA communication, consisting of transmitter, network star coupler, and receiver.

The time-to-space conversion permits us to evaluate new modulation techniques that were previously inaccessible, due to the high temporal resolution they required. An example of a possible modulation technique is pulse position modulation (PPM) (see Fig. 10). The advantage of PPM is that several information bits can be encoded on a single pulse, resulting in an efficient signaling format. The information bits are encoded at the transmitter by varying the time at which the pulse is transmitted. If we wish to encode $\mathrm{b}$ bits on each pulse, the transmitter will need to select one of $M$ transmission slots (where $M=2^{b}$ ) for the data encoded pulse. After decoding the CDMA signal at the receiver, a time-to-space conversion takes place. The transformed signal has the form of a 
bright spot at one location, corresponding to the properly decoded signal, and random low intensity light from other users distributed elsewhere. A photo-detector array, placed behind a mask with narrow slits at locations corresponding to the time delays utilized in the PPM, detects the time-to-space mapped signal. The slits perform the required femtosecond scale time filtering. Decision circuitry at the receiver selects the largest signal from the detector array, extracting the transmitted data symbol. The 'choose largest' decision logic improves the bit error rate performance, as the decision is based on $M$ statistical measurements.

(a)

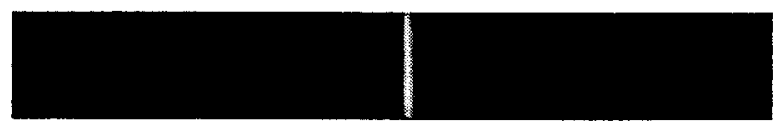

(b)

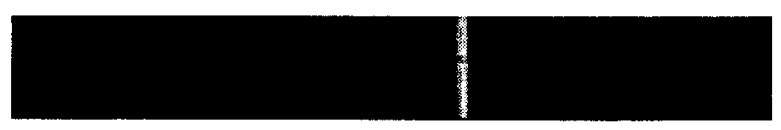

(c)

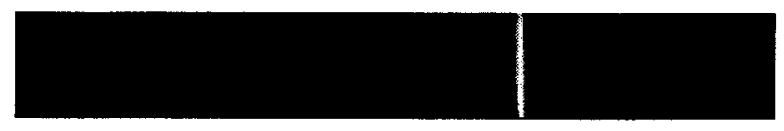

(d)

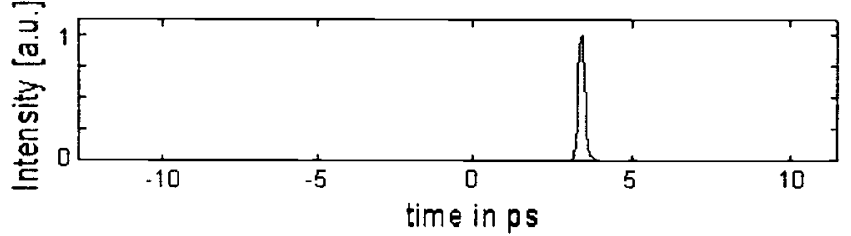

Fig. 11. Experimental results of recovered data from three different time slots using PPM/CDMA.

(a)
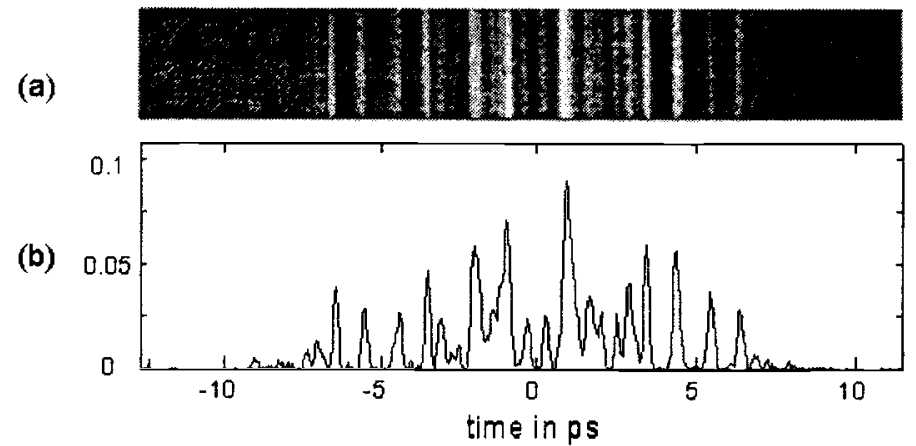

Fig. 12. (a) Experimental results of received interference from an unmatched transmitter using PPM/CDMA. The photo was enhanced for better visualization. (b) Signal values from unenhanced image.

The pulse imager at the receiver requires a reference pulse for converting the temporal signal to the space domain. As femtosecond scale time synchronization between transmitter and receiver is unfeasible, we propose to transmit the reference pulse along with the data encoded pulse ${ }^{54}$. Therefore, the information is encoded by the time difference between the two transmitted pulses. After the receiver's decoding filter, the two ultrashort pulses, with added pseudo-random background noise from other communicating members, perform the pulse imaging. This method eliminates laser pulse jitter from effecting the transmission, as the same seed pulse is used to generate the two transmitted pulses. Experimental results of PPM/CDMA modulation recovered data are shown in 
Fig. 11, where pulse images of two different time slots were chosen at the transmitter, and recovered faithfully at the receiver. Fig. 12 shows the received interference signal from an unmatched encoding/decoding CDMA pair, generating a low intensity signal distributed over a wide region.

\section{SUMMARY}

We have introduced various new optical processing techniques to achieve spatiotemporal conversion, storage, and encoding and decoding of optical short pulse signals for optical communication applications. These new methods can process 4-D information, consisting of the 3 spatial coordinates and the temporal evolution of optical signals. We have demonstrated application of photorefractive volume holographic materials for spatialtemporal signals interaction, both in the image domain as well as in the Fourier transform domain. To meet the growing operational speed requirements, we have explored the optical nonlinear wave mixing to achieve signal conversions with femtosecond scale response times. For secure communication as well as multiple user access, we have studied combining our PPM method with the optical CDMA technique, using our time imaging technique. The PPM/CDMA technique was demonstrated experimentally.

Transferring the current technologies into more practical devices requires resolving two big challenges in future research: the signal processing speed and the signal conversion efficiency. The recent discoveries of cascaded second harmonic generation and the quasi-phase-matching using periodically poled crystals, as well as composite nonlinear materials are important research directions capable of increasing the speed and the efficiency of the spatio-temporal processing, and advancing these techniques towards future practical systems.

\section{ACKNOWLEDGMENTS}

This work was supported in part by the Ballistic Missile Defense Organization, the U.S. Air Force Office of Scientific Research, NATO and the National Science Foundation. Dan Marom acknowledges the support of the Fannie and John Hertz foundation.

\section{REFERENCES}

1. R. L. Fork, C. H. Brito Cruz, P. C. Becker, and C. V. Shank, "Compression of optical pulses to six femtoseconds by using cubic phase compensation," Opt. Lett. 12, 483-485 (1987)

2. M. T. Asaki, C.-P Huang, D. Garvey, J. Zhou, H. C. Kapteyn, and M. M. Murnane," Generation of 11-fs pulses from a self-mode-locked Ti: Sapphire laser," Opt. Lett. 18, 977-979(1993)

3. P. F. Curley, Ch. Spielmann, T. Brabec, F. Krausz, E. Wintner, and A. J. Schmidt, "Operation of a femtosecond $\mathrm{Ti}$ : sapphire solitary laser in the vicinity of zero group-delay dispersion," Opt. Lett. 18, 54-56 (1993)

4. C. P. J. Barty, T. Guo, C. LeBlanc, F. Raksi, C. Rose-Petruck, J. A. Squier, K. R. Wilson, V. V.Yakovlev, and K. Yamakawa,"Generation of 18-fs, multiterawatt pulses using regenerative pulse," Opt. Lett. 21, 668-670 (1996) 
58 / Critical Reviews Vol. CR74

5. J. A. Salehi, A. M. Weiner, and J. P. Heritage, "Coherent ultrashort light pulse codedivision multiple access communication systems," J. of Lightwave Tech. 8, 478491(1990)

6. E. A. De Souza, M. C. Nuss, W. H. Knox, and D. A. B. Miller, "Wavelength division multiplexing with femtosecond pulses." Opt. Lett 20, 1166-1168(1995)

7. H. Chen, Y. Chen, D. Dilworth, E. Leith, J. Lopez, and J. Valdmanis, " Twodimensional imaging through diffusing media using 150-fs gated electronic holography techniques," Opt. Lett. 16, 487-489(1991)

8. M. R. Hee, J. A. Izatt, E. A. Swanson, and J. G. Fujimato, "Femtosecond transillumination tomography in thick tissue," Opt. Lett. 18, 1107-1109(1993)

9. B. B. Das, K. M. Yoo, and R. R. Alfano, "Ultrlfast time-gated imaging in thick tissues: a step toward optical mammography," Opt. Lett. 18, 1092-1094(1993)

10. Hanninen, P.E.; Hell, S.W.; Salo, A.J.; Soini, E.; and others., "Two-photon excitation 4Pi confocal microscope: enhanced axial resolution microscope for biological research," Appl. Phy. Lett. 66, 1698-1700(1995)

11. M. Muller, J. Squier, and G. J. Brakenhoff, " Measurement of femtosecond pulses in the focal point of a high-numerical-aperture lens by two-photon absorption," Opt. Lett. 20, 1038-1040(1995)

12. S. A. Rice,"New ideas for guiding the evolution of a quantum system," Science 258, 412-413(1992)

13. W. S. Warren, H. Rabitz, and M. Dahlen, " Coherent control of quantum dynamics: the dream is alive," Science 269, 1581-1589(1993)

14. J. Che, J. L. Krause, M. Messina, K. R. Wilson, and Y. J. Yan,"Detection and control of molecular quantum dynamics," J. Phys. Chem. 99, 14949-14958(1995)

15. A. M. Weiner, J. P. Heritage, and J. A. Salehi, "Encoding and decoding of femtosecond pulses," Opt. Lett. 13, 300 (1988).

16. J. A. Salehi, A. M. Weiner, and J. P. Heritage, " Coherent ultrashort light pulse codedivision multiple access, J. of Lightwave Technology. 8, 478-91(1990)

17. Y. Taketomi, J. E. Ford, H. Sasaki, J. Ma, Y. Fainman and S. H. Lee, "Incremental recording for photorefractive hologram multiplexing", Opt. Lett. 16, pp. 1774-1776 (1991).

18. A. Yariv, "Interpage and interpixel cross talk in orthogonal (wavelength-multiplexed) holograms," Opt. Lett., 18, 652-654 (1993)

19. K. Curtis, C. Gu, D. Psaltis, "Cross-talk in wavelength multiplexed holographic memories," Opt. Lett. 18, 1001-1003 (1993)

20. P. C. Sun, Y. Mazurenko, W.S.C Chang, P.K.L.Yu, Y. Fainman,"All-optical parallelto-serial conversion by holographic spatial-to-temporal frequency encoding, "Optics Letters, 20, 1728-1730 (1995)

21. P. C. Sun; Y. Fainman, Y. Mazurenko, D. Brady, "Space-time processing with photorefractive volume holography," (Photorefractive Fiber and Crystal Devices: Materials, Optical Properties, and Applications, San Diego, CA, USA, 12-13 July 1995). Proceedings of the SPIE - The International Society for Optical Engineering, 1995, vol.2529: 157-70. 
1999 Euro-American Workshop on Optoelectronic Information Processing / 59

22. C. Froehly, B. Colombeau, and M. Vampouille, "Shaping and analysis of picosecond light pulses, " in Progress in Optics, E. Wolf Ed. (North Holland, Amsterdam), Vol. XX, 65-153(1983)

23. A. M. Weiner, D. E. Leaird, J. S. Patel, and J. R. Wullert, "Programable femtosecond pulse shaping by use of a multielement liquird-crystal phase modulator," Opt. Lett. 15, 326-328(1990)

24. M. E. Fermann, V. da Silva, D. A. Smith, Y. Silberberg, A. M. Weiner, "Shaping of ultrashort optical pulses by using an integrated acousto-optic tunable filter," Opt. Lett. 18, 1505-1507(1993)

25. M. M. Wefers and K. A. Nelson,"Generation of high-fidelity programmable ultrafast optical waveforms," Opt. Lett. 20, 1047-1049(1995)

26. M. C. Nuss and R. L. Morrison,"Time-domain images,"Opt. Lett. 20, 740-742(1995)

27. Y. T. Mazurenko, " Holography of wave packets," Appl. Phys. B, 50, 101-113(1990)

28. A. M. Weiner, D. E. Leaird, D. H. Reitze, E. G. Paek, "Femtosecond spectral holography," IEEE J. Quantum Electron. 28, 2251-2253(1992)

29. K. Ema, M. Kuwata-Gonokami, And F. Shimizu, "All-optical sub-Tbits/s serial-toparallel conversion using excitonic giant nonlinearity," Appl. Phys. Lett. 59, 2799$2801(1990)$

30. M. C. Nuss, M. Li, T. H. Chiu, A. M. Weiner, and A. Patrovi, "Time-to-space mapping of femtosecond pulses", Opt. Lett. 19, 664-666(1994)

31. Y. Ding, R. M. Brubaker, D. D. Nolte, M. R. Melloch, and A. M. Weiner, " Femtosecond pulse shaping by dynamic holograms in photorefractive multiple quantum wells, Optics Letters 22, 718-720(1997)

32. J. S. Aitchison, Y. Silberberg, A. M. Weiner, D. E. Leaird, M. K. Oliver, J. L. Jackel, E. M., Vogel, and P. W. E. Smith, "Spatial optical solitons in planar glass waveguides," J. Opt. Soc. Am. B, 8, 1290-1297(1991)

33. R. Danielius, P. D. Trapani, A. Dubietis, A. Piskarskas, and D. Podenas, "Selfdiffraction through cascaded second-order frequency-mixing effects in b-barium borate," Opt. Lett. 18, 574-576(1993)

34. Roland Schiek, "Nonlinear refractive caused by cascaded second-order nonlinearity in optical waveguide structures," J. Opt. Soc. Am. B, 10, 1848-1855(1993)

35. G. Assanto, I. Torelli, and S. Trillo, "All-optical processing by means of vertical interactions in second-order cascading: novel approaches," Opt. Lett. 19, 17201722(1994)

36. M. A. Krumbugel, J. N. Sweeter, D N Fittinghoff, K. W. Delong, and R. Trebino, "Ultrafast optical switching by use of fully phase-matched cascaded second-order nonlinearties in a polarization gate geometry," Opt. Lett. 22, 245-247(1997)

37. M. Asobe, I. Yokohama, H. Itoh, and T. Kalio, "All-optical switching by use of cascading of phase-matched sum-frequency-generation and difference-frequency-generation processes in periodically poled LiNbO3," Opt. Lett. 22, 274-276(1997)

38. P. C. Sun, Y. Mazurenko, and Y. Fainman, "Femtosecond pulse imaging: ultrafast optical oscilloscope," Journal of Optical Society of America. A 14, 1159-1170(1997) 
$60 /$ Critical Reviews Vol. CR74

39. L. E. Myers, R. C. Eckardt, M. M. Fejer, and R. L. Byer, W. R. Bosenberg, and J. W. Pierce, "Quasi-phase-matched optical parametric oscillators in bulk periodically poled LiNbO3," J. Opt. Soc. Am. B, 12, 2102-2116(1995)

40. L. E. Myers, G. D. Miller, R. C. Eckardt, M. M. Fejer, R. L. Byer, and W. R. Bosenberg, " Quasi-phase-matched 1.064-mm-pumped optical parametric oscillator in bulk periodically poled LiNbO3," Opt. Lett. 20, 52-54 (1995)

41. P. Vidakovic, D. J. Lovering, J. A. levenson, J. Webjorn, and P. St. J. Russell, "Large nonlinear phase shift owing to cascaded $\chi^{(2)}$ in quasi-phase-matched bulk LiNbO3," Opt. Lett. 22, 277-279(1997)

42. R. W. Boyd, and J. E. Spie, "Nonlinear optical susceptibilities of layered composite material," J. Opt. Soc. Am. B, 11, 297-303(1994)

43. G. L. Fisher, R. W. Boyd, R. J. Gehr, S. A. jenekhe, J. A. Osaheni, and J. E. Spie, "Enhanced nonlinear optical response of Composite Material," Phy. Rev. Lett. 74, 18711874(1995)

44. Y. N. Denisyuk, D. I. Staselko, and R. R. Herke, "On the effect of the time and spatial coherence of radiation source on the image produced by a hologram," in Proceedings, Applications of Holography, Besancon (July, 1970)

45. D. I. Staselko, Y.N. Denisyuk, and A. G. Smirnow, "Holographic recording of the timecoherence pattern of a wave train from a pulsed laser source," Opt. Spectrosc., 26 41(1969)

46. Nils Abramson, " Light-in-flight recording by holography," Opt. Lett., 3, 121(1978)

47. K. B. Hill, K. G. Purchase, and D. J. Brady, "Pulsed-image generation and detection," Opt. Lett. 20, 1201 (1995)

48. E. Leith, H. Chen, Y. Chen, D. Dilworth, J. Lopez, R. Masri, J. Rudd, and J. Valdmanis, "Electronic Holography and speckle methods for imaging through tissue using fetosecond gated pulses," Appl. Opt. 30, 4204 (1991)

49. Yu. T. Mazurenko, "Interference of spectrally dispersed light," Opt. and Spectr. 56, 357 (1984)

50. Yu. T. Mazurenko, "Detection and reconstruction of ultrashort pulses by interference of spectrally dispersed light," Sov. J. Quan. Elec. 15, 815 (1985)

51. Yu. T. Mazurenko, S. E. Putilin, V.L. Bogdanov, G.V. Lukomskij, M.V. Smirnov and Yu. A. Cherkasov, "Spectral holography of pico- and nanosecond laser pulses," SPIE Vol. 2097 Laser Application, 380 (1993)

52. D. Psaltis, F. Mok, H. Li, "Nonvolatile storage in photorefractive crystals," Optics Letters, 1 19, 210-212 (1994).

53. H.P. Sardesai, and A.M. Weiner, "Nonlinear fibre-optic receiver for ultrashort pulse code division multiple access communications," Elec. Lett. 33, 610 (1997)

54. P. C. Sun, Y. Mazurenko, and Y. Fainman,"Real-time 1-D coherent imaging through single-mode fibers by space-time conversion processors," Opt. Lett., 22, 1861-1863 (1997) 